\title{
The interplay of dense gas and stars in M33
}

\author{
Carsten Kramer ${ }^{1}$, Christof Buchbender ${ }^{1}$, Guillermo \\ Quintana-Lacaci $^{1}$, Jonathan Braine ${ }^{2}$, Pierre Gratier ${ }^{2}$ and \\ Erik Rosolowsky ${ }^{3}$
}

${ }^{1}$ Instituto Radioastronomia Milimetrica (IRAM), Av. Divina Pastora 7, E-18012 Granada, Spain

email: kramer@iram.es

${ }^{2}$ Observatoire Bordeaux, LAB - UMR 5804, 2, rue de l'Observatoire, BP 89 -33271 Floirac Cedex - France

${ }^{3}$ University of British Columbia, 3333 University Way, Kelowna, BC V1V 1V7, Canada

\begin{abstract}
We are studying the interplay of star formation and its 'fuel', the molecular gas (diffuse and dense) at selected positions along the major axis of M33. We have observed the ground-state transitions of $\mathrm{HCN}, \mathrm{HCO}^{+}$, and ${ }^{13} \mathrm{CO}$ using the IRAM $30 \mathrm{~m}$ telescope. These data will complement existing CO, HI, Spitzer, and radio continuum maps. Furthermore, these data will be complemented by far-infrared maps of [CII], $\mathrm{H}_{2} \mathrm{O}$, [OI], [NII], and the dust continuum taken with Herschel in the open time key project HERM33ES.
\end{abstract}

M33 is a spiral galaxy located at a distance of $840 \mathrm{kpc}$. Observations of small scale structures in M33 do not suffer from distance ambiguities like galactic observations do. The IRAM- $30 \mathrm{~m}$ beam at $89 \mathrm{GHz}$ is $27^{\prime \prime}$, which corresponds to only $92 \mathrm{pc}$. M33 is seen almost face-on allowing to study individual cloud complexes.

It is the nearest late-type galaxy, is chemically and dynamically young, roughly 10 times less massive than the Milky Way and with an overall metallicity subsolar by a factor 2-3. With a well-defined metallicity gradient (Magrini et al. 2007, Rosolowsky \& Simon 2008), M33 is a particularly interesting object, filling in the metallicity gap between the Milky Way and the Magellanic Clouds. Studying metallicity effects, e.g. on the CO-to- $\mathrm{H}_{2}$ conversion factor, is also of importance for interpreting the emission of objects at high redshifts.

Due to their large dipole moments, $\mathrm{HCN}$ and $\mathrm{HCO}^{+}$trace the dense gas $\left(n>10^{4} \mathrm{~cm}^{-3}\right)$ in galaxies. And as stars condense out of the densest material, these molecules may be good tracers of the star formation rate (SFR). The role of HCN as tracer of the SFR was recently discussed by Wu et al. (2005), combining Galactic data with data of nearby galaxies and starbursts. They show that HCN is a much better tracer of star formation than CO (see also Gao \& Solomon 2004). A similar conclusion should hold for $\mathrm{HCO}^{+}$. Previous studies of the $\mathrm{HCO}^{+} / \mathrm{HCN}$ ratio find values around 1, e.g. 1.1 in M31 (Brouillet et al. 2005) or 0.5 - 1.6 in a sample of galactic nuclei, depending only slightly on environment (Krips et al. 2008).

Using the IRAM 30m telescope, we detected $\mathrm{HCO}^{+}(1-0)$ and $\mathrm{HCN}(1-0)$ at several positions along the major axis of M33 out to $4 \mathrm{kpc}$. We find rather large $\mathrm{HCO}^{+} / \mathrm{HCN}$ ratios of integrated intensities of $\sim 10$ in the central regions. In addition we find indications of a rise of $\mathrm{HCN}$ intensities with radius, while the SFR also drops with distance. More data are currently observed to secure this finding. The large $\mathrm{HCO}^{+} / \mathrm{HCN}$ ratios in the center may reflect the low $\mathrm{N} / \mathrm{H}$ abundance of $\mathrm{M} 33$. It is lower than the $\mathrm{N} / \mathrm{H}$ solar value (Asplund et al. 2005) by a factor 5 or more (Magrini et al. 2007, 2009, priv. com.). We will use models of the chemical and physical structure of photon dominated regions (Roellig et al. 2007) to confirm this speculation. 